\title{
Tissue- and stage-specific modulation of Wingless signaling by the segment polarity gene lines
}

\author{
Victor Hatini, ${ }^{1,2}$ Peter Bokor, ${ }^{1}$ Ryoko Goto-Mandeville, ${ }^{1}$ and Stephen DiNardo ${ }^{1,2,3}$ \\ ${ }^{1}$ University of Pennsylvania School of Medicine, Department of Cell and Developmental Biology, Philadelphia, \\ Pennsylvania 19104 USA
}

\begin{abstract}
Wnt signaling controls a variety of developmental programs but the mechanisms by which the same signal leads to distinct outputs remain unclear. To address this question, we identified stage-specific modulators of Wingless (Wg) signaling in the Drosophila embryonic epidermis. We show that lines (lin) is essential for Wg-dependent patterning in dorsal epidermis. lin encodes a novel protein that acts cell-autonomously, downstream or in parallel to Armadillo (Arm) and upstream of Wg-dependent target genes. Lin can accumulate in nuclei of cells signaled by $\mathrm{Wg}$, suggesting that signaling promotes entry of Lin into the nucleus, where it cooperates with Arm and Pangolin. Thus, a stage-specific modulator is used to mediate Wg signaling activity in dorsal patterning. Hedgehog $(\mathrm{Hh})$ controls half of the parasegmental pattern dorsally and antagonizes Wg function to do so. Lin can accumulate in the cytoplasm of cells signaled by $\mathrm{Hh}$, suggesting that $\mathrm{Hh}$ antagonizes Wg function by prohibiting Lin from entering the nucleus.
\end{abstract}

[Key Words: Wingless; Hedgehog; Lines; patterning; dorsal embryonic epidermis; segment polarity genes]

Received March 8, 2000; revised version accepted April 7, 2000.

An important feature of development is the precise positioning of differentiated cell types in tissues and organs. This positioning is accomplished by special cell groups that are capable of instructing fate of distant cells (Spemann 1938). Many genes involved in pattern formation have been identified by genetic analysis of cuticle patterning in Drosophila (Nüsslein-Volhard and Wieschaus 1980). These genes include a small number of ligands, their receptors, and signal transducers. Activation of a signal transduction pathway often facilitates access of a pathway-dedicated transcription factor to the nucleus, where it regulates target gene expression. Because only a few ligands are used reiteratively to pattern different tissues, their effect on target cells has to be modified by tissue- or stage-specific interactions.

The Wnt/Wg gene family encodes glycoproteins that act on different tissues as pattern-organizing ligands (Baker 1987; Cabrera et al. 1987; Rijsewijk et al. 1987). Many segment polarity genes encode functionally conserved signal transducers of the Wnt pathway. On the basis of genetic and biochemical analysis, a model of signal transduction has emerged (for review, see Dierick and Bejsovec 1999). Wg binding to the Frizzled (Fz) or Fz-2 receptors activates the pathway. Signal transduc-

\footnotetext{
${ }^{2}$ Previous address: The Rockefeller University, New York, New York 10021 USA.

${ }^{3}$ Corresponding author.

E-MAIL sdinardo@mail.med.upenn.edu; FAX (215) 898-9871.
}

tion leads to accumulation of Armadillo (Arm) in the cytoplasm. Arm translocates to the nucleus, associates with the HMG class DNA-binding protein Pangolin [Pan; also known as Drosophila T-cell factor (dTcf)] forming a transcription complex that regulates target gene expression (Behrens et al. 1996; Molenaar et al. 1996; Brunner et al. 1997; Riese et al. 1997; van de Wetering et al. 1997). It is not clear how this pathway and its pathway-dedicated transcription complex is modulated by factors that confer stage or tissue specificity.

The Wg pathway acts in several patterning processes, eliciting varied responses. In fact, even in the same tissue, Wg input elicits distinct responses at different times. For example, early in embryogenesis, Wg input consolidates parasegmental boundaries by maintaining Engrailed (En) expression in adjacent epidermal cells (DiNardo et al. 1988; Martinez-Arias et al. 1988). Late in embryogenesis, Wg input is no longer needed for En maintenance, but rather specifies cell fate. In the ventral epidermis, it specifies the smooth cuticle cell type (Baker 1988; Bejsovec and Martinez-Arias 1991; Dougan and DiNardo 1992; Noordermeer et al. 1992). It accomplishes this specification by repressing expression of genes required for denticle fate specification including veinlet (ve; also known as rhomboid), serrate, and shaven-baby (Alexandre et al. 1999; Gritzan et al. 1999; Payre et al. 1999; Wiellette and McGinnis 1999). In the dorsal epidermis, Wg specifies a fine hair cell type but the genes mediating this response are not known (Bokor and Di- 
Nardo 1996). The molecular basis for the stage-specific response to $\mathrm{Wg}$ signaling is unclear. Because two redundant receptors $\mathrm{Fz}$ and $\mathrm{Fz}-2$ mediate all Wg signaling (Bhat 1998; Kennerdell and Carthew 1998; Bhanot et al. 1999; Chen and Struhl 1999; Muller et al. 1999), specificity is not likely to be conferred by multiple receptors with distinct specificities. Thus, transducers of Wg signaling may trigger specific responses by interacting with tissuespecific regulatory proteins, and/or signal transducers activated by another pathway. One such example is the gene teashirt (tsh), which modulates Wg signaling by binding the transcription regulatory domain of Arm (Gallet et al. 1998, 1999).

One approach to understanding Wg signaling specificity is to identify genes that promote tissue- or stagespecific Wg-dependent readouts and determine how they act. Wg has a well-defined role in the development of the segmented embryonic epidermis. In dorsal epidermis, $\mathrm{Wg}$ is required to pattern half of the parasegment (Bokor and DiNardo 1996). We screened for abnormalities in this readout, as a genetic approach for isolating signal transducers of the Wg signaling pathway and factors that mediate tissue- or stage-specific responses in Wg signaling. We re-isolated mutations in the segment polarity gene lines (lin), originally identified by Nüsslein-Volhard et al. (1984) because they affect the dorsal epidermal pattern. We provide evidence that Lin is essential for late Wg signaling activity, acting downstream of Arm but upstream of Wg target genes. In addition, Lin can localize to nuclei of cells signaled by Wg. We hypothesize that Lin interacts with nuclear Wg signal transducers and confers stage specificity to the pathway. We also find that Lin localizes to the cytoplasm of cells signaled by $\mathrm{Hh}$, suggesting that $\mathrm{Hh}$ competes with Wg signaling by exporting Lin from the nucleus.

\section{Results}

lin may be a stage-specific modulator of Wingless signaling

The fly embryonic body plan is subdivided into parasegmental units. Dorsally, rows of epidermal cells adopt four distinguishable cell fates, $1^{\circ}-4^{\circ}$, depending on their position along the parasegment, generating a precise and reproducible cuticle pattern (Fig 1A). A single row of cells differentiates large, pigmented denticles, the $1^{\circ}$ fate. This row is followed by two to three cell rows producing smooth cuticle, the $2^{\circ}$ fate. The next two to three rows secrete pigmented thick hairs, the $3^{\circ}$ fate, which are shorter than $1^{\circ}$ denticles. The following seven to eight rows secrete fine hairs, the $4^{\circ}$ fate, which are longer and less pigmented than $3^{\circ}$ cells. Three to four cell rows of smooth cuticle complete the pattern. These cell types reflect alternative fate decisions made by the underlying epithelial cells. Wg and Hh inputs specify these fates and organize the pattern (Heemskerk and DiNardo 1994; Bokor and DiNardo 1996). Hh and the homeodomain protein, En, are co-expressed in stripes (gray circles in Fig. 1A; Tabata et al. 1992), the posterior row of which pro-
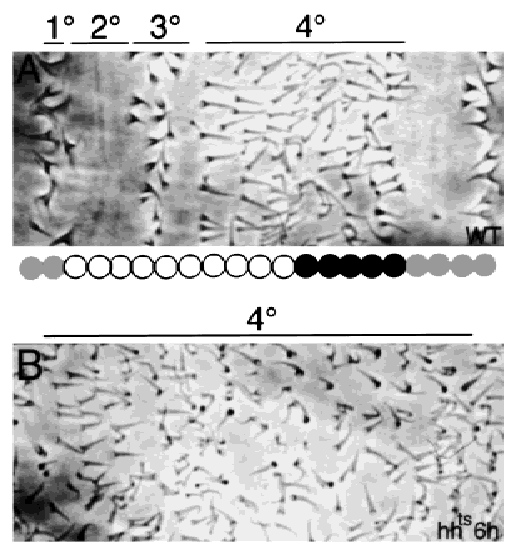

$$
1^{\circ} 1^{\circ} 2^{\circ}-3^{\circ}
$$

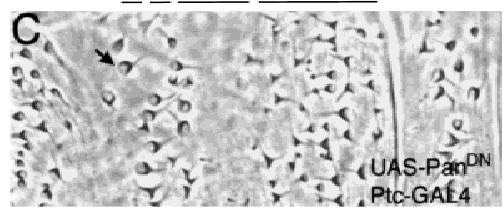

$\underline{1}^{\circ} \quad \underline{1}^{\circ} \underline{2}^{\circ} \quad 3^{\circ}$

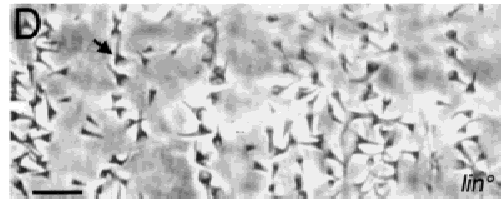

Figure 1. Contribution of $\mathrm{Hh}, \mathrm{Wg}$, and Lin to dorsal pattern. (A) Wild-type (WT) dorsal cuticle pattern. $\left(1^{\circ}, 2^{\circ}, 3^{\circ}, 4^{\circ}\right)$. Four distinct cell types visible across the parasegment. We previously mapped En/Hh (gray circles) and Wg (black circles) expression domains that underlie this cuticle pattern (Heemskerk and DiNardo 1994; Bokor and DiNardo 1996). (B) $h h^{\text {ts }}$, inactivated at 6 hr AEL. $1^{\circ}-3^{\circ}$ fates are replaced by $4^{\circ}$ fates. $(C)$ Ptc-GAL4; UAS$\mathrm{Pan}^{\mathrm{DN}}$. When $\mathrm{Wg}$ signaling is blocked, the $4^{\circ}$ fates are lost and excess $3^{\circ}$ fates develop. In addition, an ectopic $1^{\circ}$ cell row develops (arrow). (D) lin. $4^{\circ}$ fates are missing, and excess $3^{\circ}$ fates develop. In addition, an ectopic $1^{\circ}$ cell row develops (arrow). Bar, $10 \mu \mathrm{m}$ in all panels.

duces the $1^{\circ}$ fate whereas the anterior rows adopt smooth fate. $\mathrm{Hh}$ is required across half the parasegment, where cells adopt the $1^{\circ}-3^{\circ}$ fates. When late $h h$ function is blocked, the $1^{\circ}-3^{\circ}$ fates are missing and are replaced by excess $4^{\circ}$ fates (Fig. 1B; Heemskerk and DiNardo 1994). $\mathrm{Wg}$ is also required across half the parasegment, where cells adopt the $4^{\circ}$ fate. Wg is expressed anteriorly adjacent to the En/Hh domain, in a subset of the cells producing the $4^{\circ}$ cell type (black circles in Fig. 1A). To distinguish a possible role for $\mathrm{Wg}$ in specifying fate from its earlier role in maintaining En/Hh expression, we used two different conditions, each of which provides for the Wg-dependent maintenance of En/Hh but inactivates or reduces $\mathrm{Wg}$ signaling during fate specification. First, we inactivated a $w g$ temperature-sensitive allele after sufficient Wg signaling had been delivered to maintain En/ Hh [6 hr after egg laying (AEL)]. Second, we expressed dominant-negative Pan using Ptc-GAL4, which blocks $\mathrm{Wg}$ signaling only in cells flanking the En/Hh domain. 
When late Wg function is blocked in either of these ways, the $4^{\circ}$ cell fate is missing (Fig. 1C; Bokor and DiNardo 1996), and the anterior En cells adopt ectopic $1^{\circ}$ fate (arrow in Fig. 1C). These cells are flanked anteriorly by smooth cuticle. The parasegment is also narrower, suggesting cell loss. In addition, upon careful examination of these mutant embryos, occasionally we find that the domain of $3^{\circ}$ cell fates is extended. Compared with two to three rows of $3^{\circ}$ fate in wild type (Fig. 1A), five to six rows are produced in embryos blocked for $\mathrm{Wg}$ signaling (Fig. 1C). To identify additional components mediating $\mathrm{Hh}$ and $\mathrm{Wg}$ function, we screened an existing mutant collection (Nüsslein-Volhard and Wieschaus 1980). We selected the lin mutation (Fig. 1D) because the $4^{\circ}$ fate is missing, and the domain producing the $3^{\circ}$ cell fate is extended from two or three rows to five or six (Fig. 1A,D). In addition, the anterior En-expressing cells adopt $1^{\circ}$ fate instead of smooth cuticle (arrow in Fig. 1D), and smooth cuticle is produced anteriorly to these cells. Thus, the pattern in lin mutants is reminiscent of the pattern in embryos blocked for late Wg signaling (Fig. 1, cf. C and D), suggesting a role for lin in the Wg pathway.

Because Wg expression decays in lin mutants, we questioned whether this event could explain the pattern defects. However, Wg expression decays at $10 \mathrm{hr}$ AEL (see Fig. 5C, below; van den Heuvel et al. 1993), while Wg activity is required earlier, between 6 and 9 hr AEL, for dorsal patterning (Bokor and DiNardo 1996). Nevertheless, we tested directly whether restoring $\mathrm{Wg}$, by driving UAS-Wg using Arm-GAL4 in lin mutant embryos can restore dorsal patterning, and it did not (not shown).
As expected, activating Wg signaling in wild-type embryos affected the pattern (see below and Fig. 3B). Thus, $\mathrm{lin}$ is required downstream of $\mathrm{Wg}$ expression.

The pattern defect in dorsal epidermis suggests that lin mutations affect only late-stage Wg signaling. Because a maternal contribution of lin could mask an earlier role in Wg signaling, we examined embryos that lack both maternal and zygotic lin activity. However, the cuticle phenotype of these embryos is indistinguishable from zygotic lin mutants (not shown). Thus, lin is necessary only for late, Wg-dependent cell-type specification in dorsal embryonic epidermis.

In contrast, in the ventral epidermis, the major lin mutant phenotype does not reflect a defect in Wg signaling. In wild type, the denticle belt is composed of six rows, each with distinct shape, size, and polarity (Fig. 2B). In lin mutants, the first three rows are replaced with smooth cuticle (Fig. 2C). Normally, patterning in this portion of the denticle belt is controlled by epidermal growth factor-receptor (EGF-R) and not Wg signaling (O' Keefe et al. 1997; Szüts et al. 1997; Gritzan at al. 1999; Sanson et al. 1999|. Thus, the ventral pattern defect in lin mutants suggest a role for Lin within the EGF-R pathway (V. Hatini and S. DiNardo, in prep.). Note also that EGF-R function plays no role in patterning the dorsal epidermis, where we find that lin function is linked with Wg signaling. Thus, while in some tissues, lin may cooperate with a different signaling pathway, in dorsal embryonic epidermis, lin is specifically necessary for late, Wg-dependent cell-type specification. Thus, we hypothesize that lin encodes a factor necessary for stagespecific, Wg-dependent decisions. To test this hypoth-

\section{A}

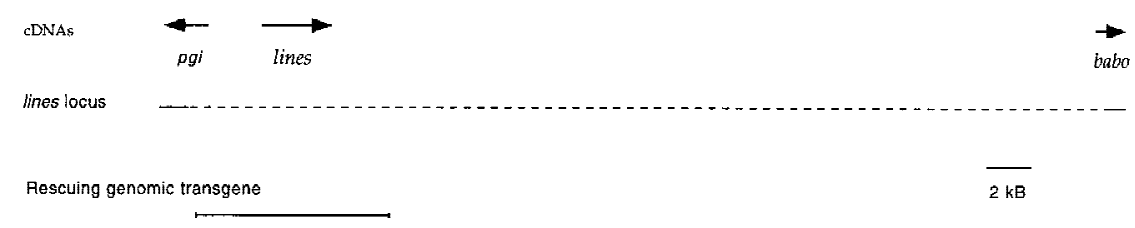

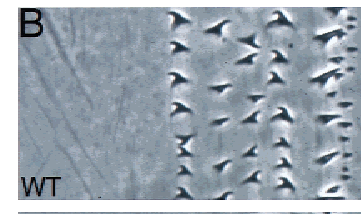
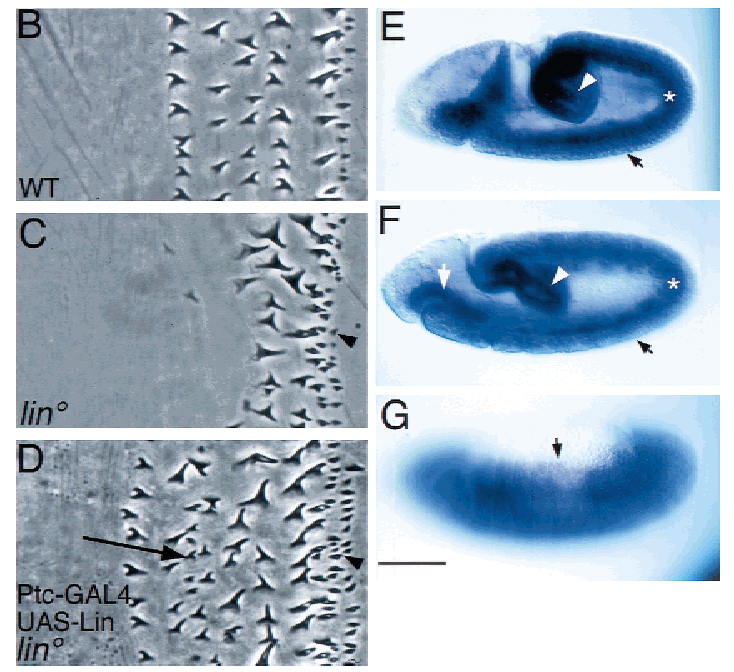

Figure 2. Rescue of lin mutants and lin mRNA expression. (A) Smallest deficiency $[\mathrm{Df}(2 \mathrm{R}) \mathrm{P} 15$; broken line] that uncovers lin and the position of the lin gene. (B) Wild-type ventral denticle belt composed of six denticle rows. (C) lin. Anteriormost three denticle rows are replaced with smooth cuticle, and denticle row 4 appears large like row 5 . Excess denticles are produced posterior to the denticle belt (arrowhead), a phenotype similar to that obtained when late Wg function is attenuated (Bejsovec and Martinez-Arias 1991). (D) lin Ptc-GAL4; UAS-Lin. Anterior denticle rows are restored, and excess row $2 / 3$ denticles are produced (arrow). Note that excess denticles posterior to the belt (arrowhead) are not restored to smooth cuticle. (E-G) RNA in situ. Low levels of lin are ubiquitously expressed in epidermis (closed arrows), with higher levels in hindgut (open arrowhead) and foregut (open arrow) and in mesoderm (asterisk). Bar, $5 \mu \mathrm{m}$ in $B-D ; 100 \mu \mathrm{m}$ in $E-G$. 
esis, we cloned the gene and analyzed its interaction with Wg signaling dorsally.

\section{Cloning of lin}

First, we mapped lin to a small deficiency, cloned all the DNA spanning the deficiency, and identified transcripts encoded in this region (Fig. 2). We constructed several overlapping genomic transgenes covering the deficiency, one of which rescued lin mutants to adulthood (Fig. 2A). This transgene spanned the complete coding region of only one cDNA. Expression of a representative cDNA encoded by this genomic fragment by use of the GAL4UAS system and the Ptc-GAL4 driver rescued ventral (Fig. 2C), as well as restored dorsal, Wg-dependent cell fates in lin mutant embryos. We identified a nonsense mutation (due to a $\mathrm{C}$ to $\mathrm{T}$ substitution) in $7 \mathrm{in}^{2 \mathrm{f}}$, which truncates the protein at position 108 of the 858-amino-acid long protein, likely generating a null allele. We have used $\operatorname{lin}^{2 \mathrm{f}}$ and $\operatorname{lin}{ }^{\mathrm{G} 2}$, which is phenotypically as strong as $7 i n^{2 \mathrm{f}}$, in the experiments described below. lin encodes a novel protein; the only notable feature is a highly acidic aminoterminal region. The lin gene produces a single mRNA species, which is expressed broadly, at low levels in the embryonic epidermis during the time of epidermal patterning (Fig. 2 E-G) and at higher levels elsewhere in the embryo (V. Hatini, P. Bokor, and S. DiNardo, unpubl.).

\section{Lin and Wg are similarly required for patterning of the dorsal epidermis}

Lin and late $\mathrm{Wg}$ activities are required for $4^{\circ}$ but not $1^{\circ}-3^{\circ}$ cell fates. To determine whether Lin and late $\mathrm{Wg}$ function are sufficient for specifying the $4^{\circ}$ cell fate, we used Arm-GAL4 to drive ubiquitous expression of Lin, $\mathrm{Wg}$, or activated Wg signal transducers. Expression of Wg or activation of the Wg pathway globally by driving activated Arm expression leads to the replacement of $1^{\circ}-3^{\circ}$ cell fates with $4^{\circ}$ fates (Fig. 3B). Similarly, driving of global Lin expression elicits an identical response, replacing the $1^{\circ}-3^{\circ}$ fates with $4^{\circ}$ fates (Fig. $3 \mathrm{C}$ ). Thus, Wg and Lin are sufficient for specifying the $4^{\circ}$ cell fate when provided at higher levels. We note that one portion of the parasegment is less sensitive to ectopic Lin or Wg activity. Driving of Lin or Wg at high levels in cells posterior to the En domain, by use of Ptc-GAL4, is sometimes not sufficient to transform the $2^{\circ}$ fate to $4^{\circ}$ (Fig. 3D). This domain is where $\mathrm{Hh}$ activity is normally highest, suggesting that $\mathrm{Hh}$ signaling competes with Lin and $\mathrm{Wg}$ in fate selection.

Because both loss and gain of Lin and Wg function exhibit similar cuticle phenotypes, we hypothesize that they act along the same or parallel pathways to specify $4^{\circ}$ fates.

Lin acts cell-autonomously in specifying the $4^{\circ}$ cell fate

Lin may contribute to Wg signaling either by controlling the production of a signal or by transducing a signal. We

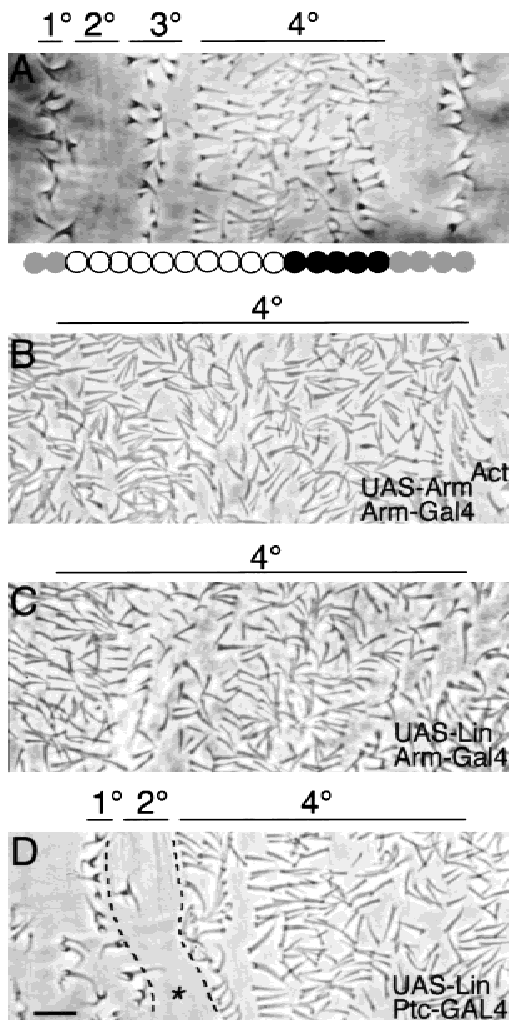

Figure 3. $\mathrm{Wg}$ and Lin are sufficient for specifying the $4^{\circ}$ cell fate. (A) Wild type. (B) UAS-Arm ${ }^{\mathrm{S} 10}$; Arm-GAL4. Activation of Wg signaling broadly shows replacement of $1^{\circ}-3^{\circ}$ fates by excess $4^{\circ}$ fates. (C) UAS-Lin Arm-GAL4. Ubiquitous overexpression of Lin also leads to replacement of $1^{\circ}-3^{\circ}$ cell fates with the $4^{\circ}$ fates. (D) Ptc-GAL4; UAS-Lin. Occasionally, overexpression of Lin is not sufficient to specify $4^{\circ}$ fate in place of $2^{\circ}$ (asterisk). Bar, 10 $\mu \mathrm{m}$ in all panels.

can discriminate between these possibilities because if Lin acts by producing a signal, restoration of restricted expression of Lin in lin mutants will restore the $4^{\circ}$ cell fate non-autonomously. If Lin is required for transducing the signal, the $4^{\circ}$ fate will be restored autonomously only in cells expressing Lin. In lin mutants, the anterior- and posterior-most En rows differentiate the $1^{\circ}$ denticle type, while two to three cell rows internal to the En domain differentiate smooth cuticle (Fig. 4A). Use of En-GAL4 and UAS-Lin to restore Lin in the En domain transforms all the En-expressing cells cell-autonomously to the $4^{\circ}$ cell fate (Fig. 4B). Cells flanking the En domain are not affected. Similarly, restoration of Lin in the $\mathrm{Wg}$ domain in lin mutants by use of Wg-GAL4 restores the $4^{\circ}$ fate (Fig. 4D), but only in that portion of the $4^{\circ}$ field that expresses Wg. Two to three rows of cells anterior to the Wg domain still adopt $3^{\circ}$ fate (arrowheads in Fig. 4C). Restoration of Lin activity in Wg-producing cells does not restore the full pattern. Thus, these two experiments indicate that Lin generally does not regulate expression of a signal, but rather acts cell-autonomously to specify the $4^{\circ}$ cell fate.

There is one instance where Lin may regulate a signal. 
Figure 4. lin acts cell-autonomously. (A) lin EnGAL4; UAS-LacZ. Activity stain reveals the En domain in lin mutants (enclosed by broken lines), which includes ectopic and normal $1^{\circ}$ cell types and two to three rows of smooth cuticle in between. (B) Expression of UAS-Lin with EnGAL4 in lin mutants transforms all cells in the En domain into $4^{\circ}$ cells. (C) Wild type. Two to three rows differentiate as $3^{\circ}$ cells (arrowheads). $(D)$ Expression of UAS-Lin with Wg-GAL4 in lin mutants restores $4^{\circ}$ fate to five to six cells rows where $\mathrm{Wg}$ is expressed, but not more anteriorly. (Arrowheads) Five rows that still differentiate as $3^{\circ}$ cells. Note that the ectopic $1^{\circ}$ cell type is restored to smooth cuticle. Bar, $10 \mu \mathrm{m}$ in all panels.
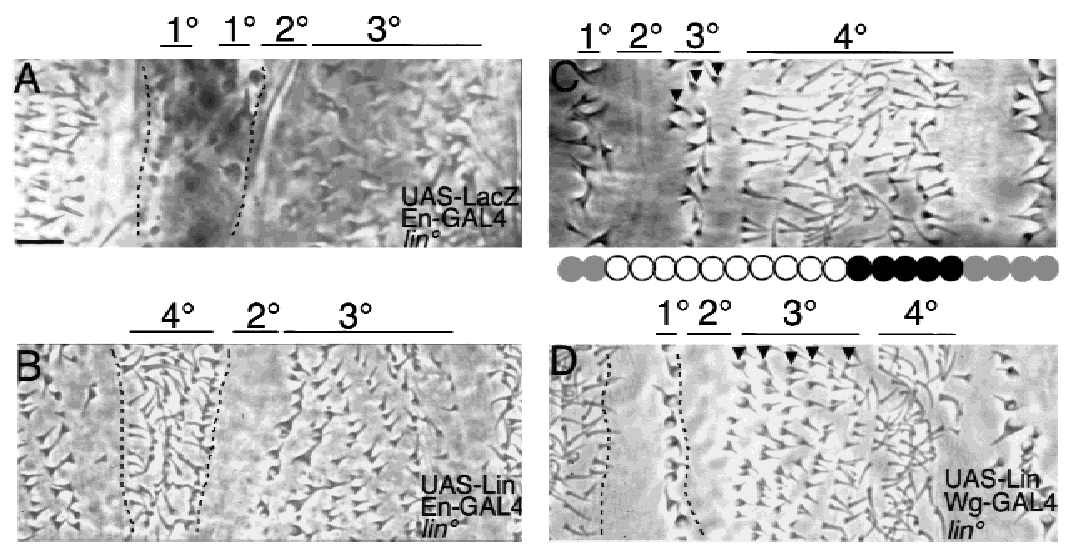

Expression of Lin in the Wg domain rescues the ectopic $1^{\circ}$ denticle row found in the En domain of lin mutants (Fig. 4D). Thus, Lin has a second role within Wg-expressing cells, and, in this case, it acts non-autonomously to specify the (smooth) fate of the posteriorly adjacent row of En cells.

\section{Lin is necessary for Wg target gene regulation}

The suggestion that Lin acts in some way to specify dorsal cell fate has so far relied on cuticle analysis, which represents the final differentiated state of the cells, first visible at about $13 \mathrm{hr}$ AEL. However, Wg signaling specifies these cell fates between 6 and 9 hr AEL (Bokor and DiNardo 1996). Thus, to test whether or not Lin acts in concert with $\mathrm{Wg}$, we need to identify earlier molecular markers for Wg patterning and test whether Lin activity affects these markers. The first $\mathrm{Wg}$-dependent target gene is $\mathrm{wg}$ itself. If $\mathrm{Wg}$ function is blocked late by expression of dominant-negative Pan with the Ptc-GAL4 driver, late $w g$ expression is lost from both the dorsal and the ventral epidermis (Fig. 5B). Reciprocally, if Wg signaling is activated by driving of activated Arm expression with the Ptc-GAL4 driver, an ectopic Wg stripe is induced posterior to the En domain (Fig 5E). Thus, wg gene expression depends on Wg input and provides a molecular readout for the pathway. In lin mutants, late wg expression fades from the dorsal epidermis of fully retracted embryos (10 hr AEL, Fig. 5C; van den Heuvel et al. 1993; Bokor and DiNardo 1996). Reciprocally, overexpression of Lin using the Ptc-GAL4 driver activates wg expression posterior to the En domain in the dorsal epidermis (Fig 5F). The ectopic expression of $\mathrm{wg}$ is identical to that obtained by expression of activated Arm posterior to the En domain. The only distinction is that the effect of Lin is restricted to the dorsal epidermis, while global activation of Wg signaling affects the ventral epidermis as well (Fig. 5, cf. E and F). Thus, Wg input and Lin are both necessary and sufficient for activation of $w g$ gene expression in dorsal epidermis.

The second Wg-dependent target gene is ve, which is expressed in a row of cells posteriorly adjacent to the
Figure 5. Lin and $\mathrm{Wg}$ similarly control wg gene expression. (A) Wild-type, stage 13. Wg protein is expressed in stripes in both dorsal (open arrowhead) and ventral (open arrow) epidermis. (B) In embryos blocked for late Wg signaling, Wg expression is lost ventrally and is fading dorsally. (C) lin. Wg expression is maintained ventrally, but lost dorsally. In both $B$ and $C$, $\mathrm{Wg}$ protein is missing from most dorsal cells, but expression is up-regulated in a few individual cells. (D) Wild type, Wg RNA (blue), En protein (brown). $w g$ is expressed anterior to the En domain. (E) In embryos overexpressing activated Arm, an ectopic $w g$ stripe is induced posterior to the En domain ventrally (arrows) and dorsally (arrowheads). $(F)$ In embryos overexpressing Lin, an ectopic stripe of $\mathrm{wg}$ is induced posterior to the En domain, but only dorsally (arrowhead). Bar, $50 \mu \mathrm{m}$ in $A-C$; $10 \mu \mathrm{m}$ in $D-F$.
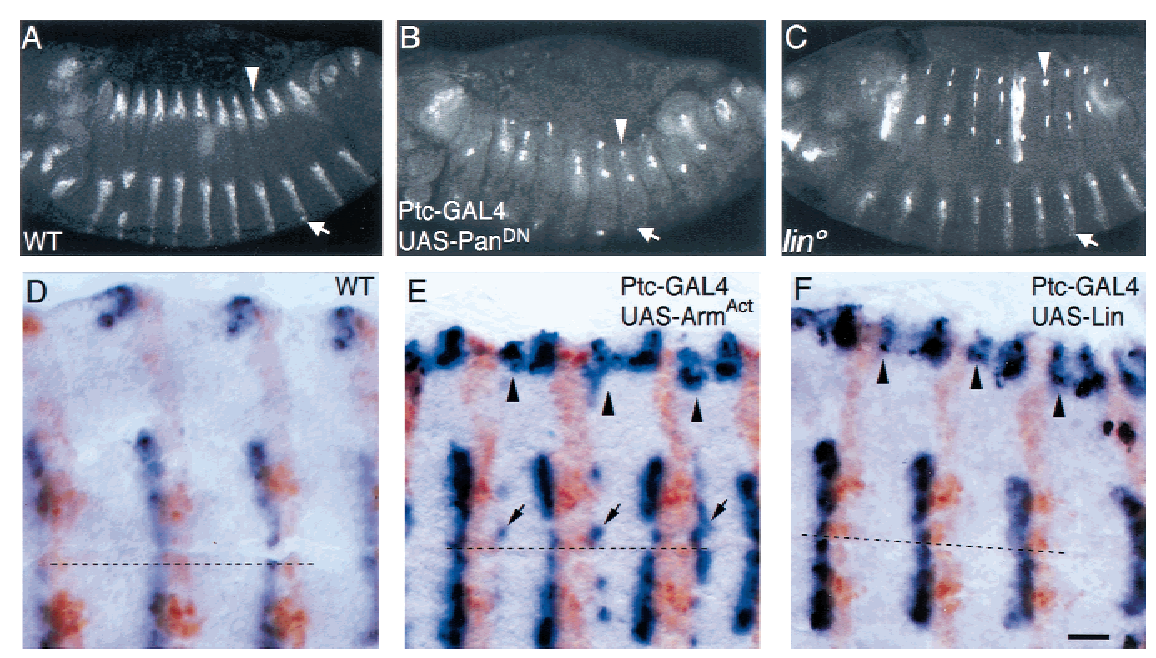
En/Hh-expressing cells (Fig. 6A; Bier et al. 1990). This spatially restricted pattern is regulated in ventral epidermis by Wg signaling (Alexandre et al. 1999; Gritzan et al. 1999). We now show that $\mathrm{Wg}$ regulates ve expression dorsally as well. For example, if Wg function is inactivated at late stages, ve is ectopically expressed anterior to the En domain (Fig. 6B). Reciprocally, if the Wg pathway is broadly activated, ve expression is repressed (Fig. $6 \mathrm{C})$. Thus, ve expression also provides a molecular readout for the Wg pathway. In lin mutant embryos, a second stripe of $v e$ is induced anterior to the $\mathrm{En} / \mathrm{Hh}$ cells in the dorsal epidermis (Fig. 6D). Thus, Lin and Wg function are similarly required to repress ve gene expression.

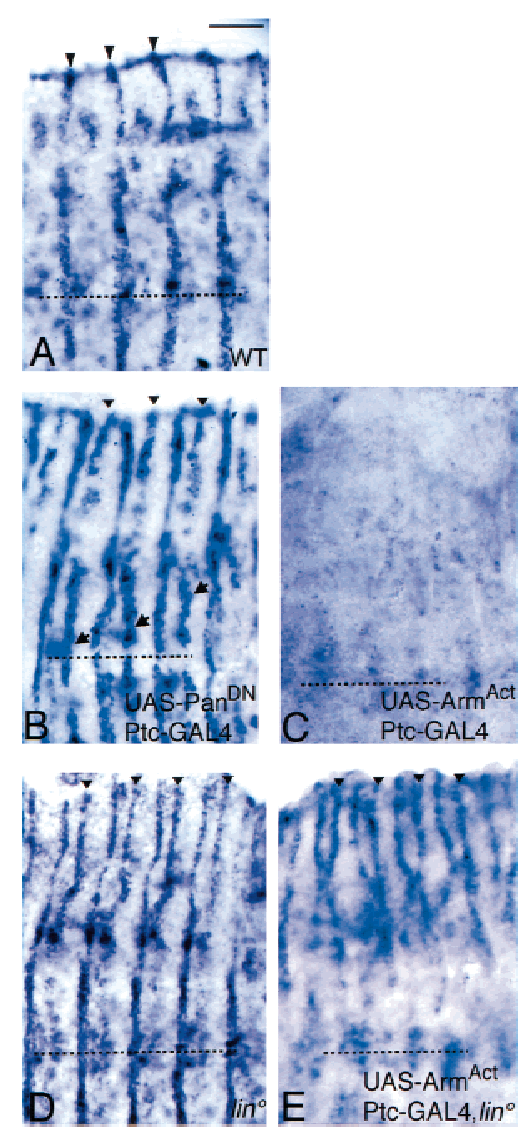

Figure 6. Lin and Wg similarly control ve gene expression. $(A)$ Wild type, Ve RNA in situ. ve is expressed in one epidermal stripe extending from the dorsal edge (arrowheads) to the ventral midline (broken line). $v e$ is expressed posteriorly adjacent to the En domain (Gritzan et al. 1999). Also note ve expression in some mesodermal and nerve cells underlying the epidermis. (B) Blockage of Wg signaling activity by expression of dominant-negative Pan with the Ptc-GAL4 driver leads to an ectopic stripe of ve that extends from the dorsal edge (arrowheads) to the ventral midline (arrows). The ectopic ve stripe is anteriorly adjacent to the En domain (Gritzan et al. 1999). (C) Reciprocally, activation of the Wg pathway by expression of activated Arm with the Ptc-GAL4 driver leads to repression of ve expression. (D) In lin mutants, an ectopic stripe of ve is found dorsally (arrowheads), but not ventrally. $(E)$ Activation of $\mathrm{Wg}$ signaling by expression of activated Arm, with the Ptc-GAL4, in lin mutants represses ve expression ventrally but not dorsally. Bar, $20 \mu \mathrm{m}$ in all panels.
We conclude that Lin acts in concert with $\mathrm{Wg}$ in regulating target genes and consequently patterning the dorsal cell types.

\section{Lin is necessary for Wg signaling in the dorsal epidermis}

Because Lin acts cell-autonomously upstream of Wg target genes, we determined where along the Wg signaling pathway Lin function is required. We accomplished this determination by forcing activation of cytoplasmic Wg signal transducers in lin mutants and testing cuticle pattern and ve gene regulation. We activated Wg signaling by overexpressing Dishevelled (not shown) or using constitutively activated Arm, with the UAS-GAL4 system (Figs. 6E and 7C). Activation of Wg signaling in wild-type embryos leads to global specification of the $4^{\circ}$ cell fate across the dorsal epidermis. In contrast, this specification does not occur if lin function is removed (Fig. 7, cf. $\mathrm{B}$ and $\mathrm{C}$ ). In fact, the pattern resembles the lin mutant pattern. For example, rather than adopting $4^{\circ}$ fate, $1^{\circ}$ and $2^{\circ}$ fates are still established, as is the ectopic $1^{\circ}$ fate and the smooth fates anterior to these cells (Fig. 7C). Cells in the remainder of the parasegment resemble immature $3^{\circ}$ rather than $4^{\circ}$ cells. For example, the cuticle protrusions are shorter and more pigmented than $4^{\circ}$ cells, but their base is narrower than fully mature $3^{\circ}$ cells. The expression of a molecular marker is consistent with the cuticle pattern, because now ve is not repressed by increased $\mathrm{Wg}$ signaling if lin activity is removed (Fig. 6E). Note that ventrally, Wg activation still represses ve even when lin activity is removed, further demonstrating the restriction of the role of Lin to dorsal Wg signaling. Thus, in dorsal epidermis, Lin is crucial for completing Wg signal transduction, acting downstream or in parallel to Arm.

Note the epistasis also is consistent with Lin being a downstream target gene of the Wg pathway. However, this model is unlikely as lin is still expressed in embryos blocked for Wg signaling, and lin expression is not upregulated in embryos globally activated for Wg signaling (data not shown). Therefore, Lin could act either in a parallel pathway to the Wg pathway, or may cooperate with Wg signal transducers.

If Lin is acting strictly in parallel to Arm, then both Wg input and Lin must act for normal patterning. If, however, Lin is required downstream of Wg signaling then overexpression of Lin in embryos blocked for Wg signaling will bypass the need for $\mathrm{Wg}$ input and restore Wg-dependent readouts. The dorsal epidermis in embryos null for Wg signaling is poorly differentiated and occasionally missing. Expression of Lin in these embryos does not rescue the pattern (not shown). Because lin is not needed for early Wg signaling perhaps it is not surprising that Lin is not capable of restoring En expression in the absence of Wg. Expression of Lin in embryos blocked for late Wg signaling may not allow us to distinguish between the two models posed above because these embryos might contain residual Wg signaling activity. To examine this issue, we overexpressed Lin using Ptc-GAL4 in $w^{\text {ts }}$ embryos shifted to the nonpermis- 
Figure 7. Lin is essential for Wg signaling and can promote $\mathrm{Wg}$-dependent readouts when $\mathrm{Wg}$ signaling is deficient $(A)$ lin. $(B)$ Activation of Wg signaling results in global specification of $4^{\circ}$ cell types. (C) However, activation of Wg signaling in lin mutants, by expression of activated Arm with ArmGAL4, does not lead to global specification of $4^{\circ}$ cell types. Note that the ectopic $1^{\circ}$ row is preceded by a region of differentiating smooth cuticle (asterisk), as in $A .(D)$ Pattern in embryos blocked for late Wg signaling. (E) Ptc-GAL4; UAS-Lin. Excess rows of $4^{\circ}$ cells. $(F)$ Overexpression of Lin in embryos deficient in Wg signaling leads to differentiation of the $4^{\circ}$ cell type, in place of the $3^{\circ}$, in cells flanking the En domain. Note that the ectopic $1^{\circ}$ cell row is not rescued. However, anterior to this region, smooth cuticle is replaced with the $4^{\circ}$ (asterisk). Bar, $10 \mu \mathrm{m}$ in all panels.
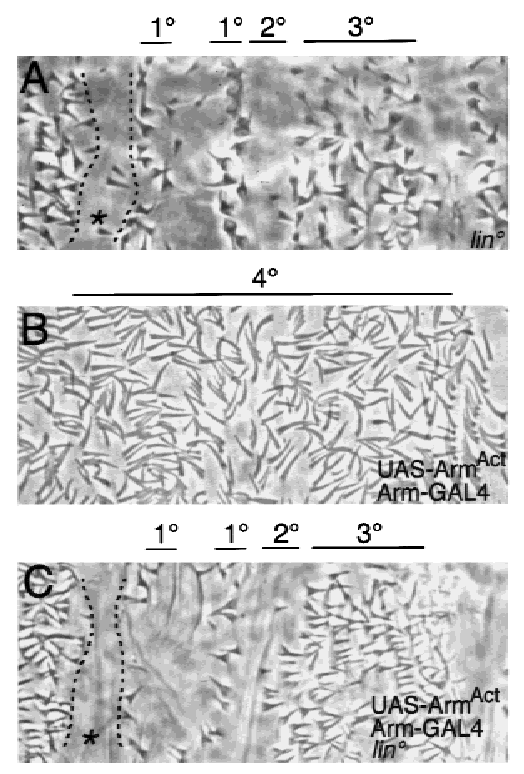
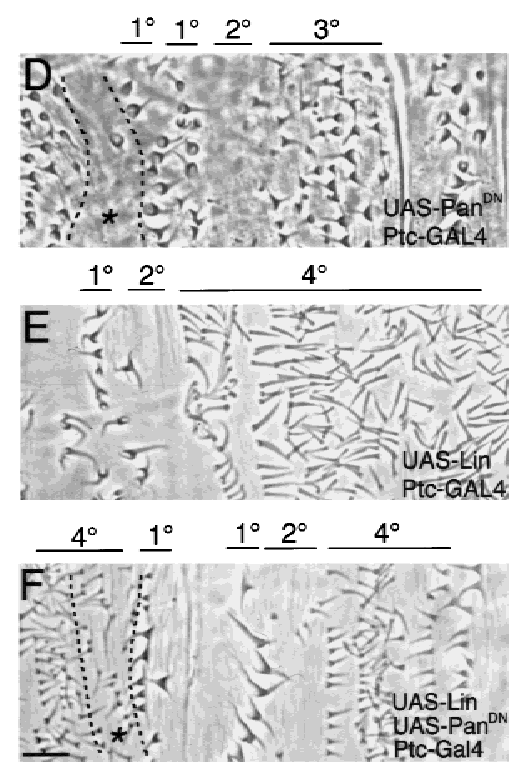

sive temperature at $6 \mathrm{hr}$ AEL (not shown), or in embryos co-expressing Lines and dominant-negative Pan, and examined their cuticle pattern. In either experiment, the usual effect on patterning caused by the defect in $\mathrm{Wg}$ signaling is blocked in embryos co-expressing Lin as the $4^{\circ}$ cell fate is restored (Fig. 7, cf. E and F). For example, anterior to the En domain, smooth cuticle is replaced with the $4^{\circ}$ type (asterisk in Fig. 7F). In addition, the $4^{\circ}$ cell fate is restored posterior to the $2^{\circ}$ domain. Expression of Lin in embryos expressing dominant-negative Pan with the ubiquitous Arm-GAL4 driver replaces all cell fates with $4^{\circ}$ cell fate (not shown). This result may have two interpretations. High levels of Lin may trigger a response independently of $\mathrm{Wg}$ input. Alternatively, any residual Wg signaling that may be present in these embryos allows Lin to function.

\section{Lin is nuclear in cells dependent on Lin and $\mathrm{Wg}$} function

In the current model for Wg signaling, Arm regulates Wg target gene expression in association with the DNAbinding protein Pan. Because our epistasis shows that Lin regulates $\mathrm{Wg}$ target gene expression downstream of Arm, we expected to find Lin protein in nuclei. Crude and purified polyclonal antisera weakly detected Lin protein by Western blot from embryonic extracts (not shown), but these sera failed to detect the protein in situ. We also modified the lin genomic rescue transgene to include a Myc tag at the carboxyl terminus of the Lin protein. The modified transgene rescued lin mutants to adulthood; however, we have not been able to detect the Myc-tagged Lin protein in situ. We conclude that Lin is expressed below the detection levels of both our anti-Lin and the commercial anti-Myc antibodies. However, Lin protein can be detected by our antibodies in situ in embryos overexpressing Lin by use of the UAS-GAL4 sys- tem (Fig. 8). For instance, embryos carrying UAS-Lin and the Ptc-GAL4 driver show the expected high levels of Lin protein both anterior and posterior to the En expression domain at $10 \mathrm{hr}$ AEL (Fig. 8A). However, Lin exhibits a different subcellular localization in each expression domain. Lin is nuclear in cells anterior to the En domain (arrowhead), in exactly those cells dependent on Lin and Wg function. Lin is preferentially cytoplasmic posterior to the En domain (arrow), which may help explain why excess Lin sometimes does not alter patterning in this domain (Fig. 3D). However, we must assume that low levels of Lin do enter the nucleus, as sometimes these cells are transformed to the $4^{\circ}$ fate. Expression of Lin with Arm-GAL4 shows that Lin is nuclear in half of the parasegment anterior to the En domain, and cytoplasmic in the other half posterior to the En domain.

To determine whether the differential localization is signal-dependent we co-expressed Lin with Wg to ectopically activate Wg signaling. In this case, Lin accumulates exclusively in nuclei both anterior (arrowhead) and posterior (arrow) to the En domain (Fig. 8B). To decrease Wg signaling, we expressed dominant-negative Pan with Ptc-GAL4. As we showed above (Fig. 5B), due to feedback regulation, this expression leads to reduced Wg expression, and therefore reduced Wg signaling. In such embryos, the differential localization of Lin is altered, but mostly at lateral positions in the embryo, where Lin occasionally localizes to the cytoplasm anterior to the En domain (small arrowheads). In the dorsal epidermis, Lin is still nuclear, which is consistent with the fact that although Pan function is compromised in these embryos, excess Lin still promotes the $4^{\circ}$ fate (Fig. $7 F$ ).

We conclude that Wg signaling can influence the subcellular localization of Lin. In addition, the nuclear localization of Lin supports our genetic model that Lin is needed to complete transduction of the Wg signal, probably by cooperating with Arm/Pan in the nucleus. Interestingly, in the ventral epidermis, where Lin is required 


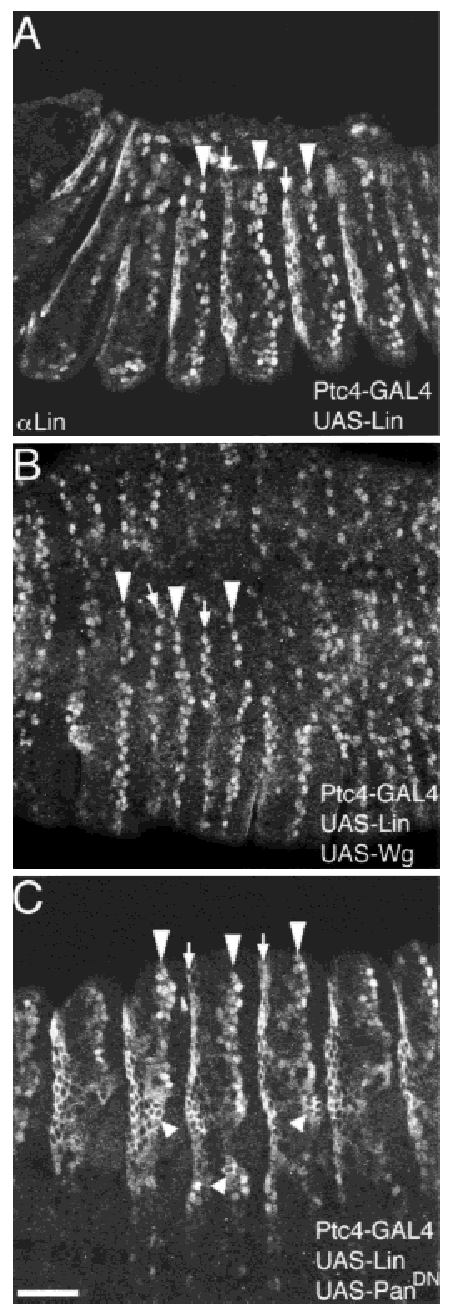

Figure 8. Differential subcellular localization of Lin. (A) PtcGAL4; UAS-Lin. Anterior to the En domain, Lin localizes to nuclei (open arrowheads), whereas posterior to the En domain, Lin localizes to the cytoplasm (open arrows). (B) Coexpression of Lin and Wg with the Ptc-GAL4 driver leads to the localization of Lin in nuclei both anterior and posterior to the En domain. $(C)$ Coexpression of Lin and dominant-negative Pan with the PtcGAL4 driver leads to the localization of Lin to the cytoplasm anterior to the En domain in lateral epidermis (small open arrowheads). Dorsally, the differential localization of Lin is maintained. Bar, $50 \mu \mathrm{m}$ in all panels.

on either side of the En domain, Lin is nuclear both anterior and posterior to that domain (not shown). Thus, Lin subcellular localization is correlated with its spatial requirement.

\section{Discussion}

We have found that lin function is absolutely essential for tissue- and stage-specific Wg signaling events. On the basis of the results of genetic tests involving both loss and gain of function and on the signal-dependent differential subcellular localization of Lin protein across the parasegment, we discuss how this novel protein may me- diate Wg-dependent cell-fate specification. More generally, we also discuss how Lin affects the balance of $\mathrm{Wg}$ and $\mathrm{Hh}$ signaling and assures proper patterning of the parasegment dorsally.

\section{Stage-specific modulators of Wg signaling}

Because Lin is not required for the early role of $\mathrm{Wg}$ signaling, its requirement for late Wg-dependent cell-type specification is stage specific. Moreover, because Lin is required only minimally for late signaling ventrally, but is essential dorsally, its role in Wg-dependent cell-type specification is tissue specific, or specific to a subregion within a tissue.

Recently another factor has been described as a stagespecific modulator of $\mathrm{Wg}$ signaling. Teashirt (Tsh), a zinc-finger protein, is necessary for late, but not early, Wg signaling in the ventral epidermis (Gallet et al. 1998, 1999). The role of Tsh has not been investigated dorsally. Although Tsh binds to the carboxyl terminus of Arm, the precise manner in which this binding allows Wg signaling is not understood. Regardless of the actual mechanism, the fact that two modulators of Wg signaling have now been described for just embryonic epidermal $\mathrm{Wg}$ function suggests that such factors will be identified for many of the other Wnt/Wg-dependent specification events throughout development. These modulators may be essential for selecting the proper tissue-specific developmental programs in response to Wnt/Wg signaling input.

Aside from Tsh and Lin being stage-specific modulators of Wg-dependent cell-type specification, the other intriguing parallel between these two genes is that both also affect the function of Homeotic proteins (de Zulueta et al. 1994; Castelli-Gair 1998). The mechanism by which each protein affects Homeotic function is unknown, although Tsh, a zinc-finger protein, and Lin, a pioneer protein, are likely to act in distinct manners biochemically. Nevertheless, the fact that both Lin and Tsh affect Wg signaling as well as Homeotic function demonstrates that these proteins are used at developmentally different times and places to modulate the effects of different factors. Note also that we have shown that lin activity has a major role in ventral epidermal patterning, but instead of affecting Wg-dependent cell types, it is necessary for those cell types dependent on EGF-R activation (V. Hatini, P. Bokor, and S. DiNardo, unpubl.; O' Keefe et al. 1997; Szüts et al. 1997).

\section{Models of lin interaction with the Wg signaling pathway}

We considered the possibility that Lin merely facilitates Wg signaling activity by increasing the efficiency of some step along the Wg pathway. However, we found that excess Wg produced in lin mutant embryos did not restore $\mathrm{Wg}$-dependent readouts, demonstrating that lin is essential for $\mathrm{Wg}$ signal transduction. In addition, we 
showed that lin activity is, in general, required cell-autonomously, indicating a role for Lin in cells receiving Wg input. Epistasis analysis helped place the requirement for lin activity relative to that for $\mathrm{Wg}$ signal transducers.

Overexpression of Lin in embryos deficient for Wg signaling (because they express a dominant-negative form of Pan) restored patterning. There are two possible explanations for this. The lin gene might be induced by $\mathrm{Wg}$ signaling and be a target of the pathway. However, lin expression is independent of $\mathrm{Wg}$ input. The second explanation relies on the likelihood that there is residual Pan (and activated Arm) activity still present in these embryos. We favor the possibility that overexpressed Lin cooperates with residual $\mathrm{Wg}$ signal transduction to specify cell fates. This model is supported by our analysis showing that Lin acts downstream of activated Arm but upstream of the expression of target genes.

Because Lin encodes a novel protein, its sequence did not yield clues for the manner in which it cooperates with Arm and/or Pan. However, we find it compelling that Lin protein accumulates in nuclei of cells in the dorsal epidermis signaled by Wg. Although with our current reagents, we can only visualize the Lin protein when overexpressed, in the very same embryos, Lin protein remains in the cytoplasm of cells signaled by $\mathrm{Hh}$. These observations, along with the results summarized above from epistasis tests, lead to three models for Lin function in Wg-dependent cell-fate specification.

First, because Lin protein is nuclear in cells in which it is acting, Lin may directly regulate the transcription of Wg target genes by interacting with DNA or DNA-bound proteins. For instance, Arm and Pan may act at one site on target genes, while Lin acts directly at an adjacent site. Because Lin does not have an obvious DNA-binding domain, it may act in combination with another DNAbinding protein. Pan bound to one site could easily be imagined to modulate the function of Lin at a second site, as LEF1/TCF proteins related to Pan can play an architectural role, regulating the assembly of multiprotein enhancer complexes (Giese et al. 1992). This scenario is reminiscent of the midgut Ubx enhancer, which contains a Wg-responsive Lef-1 DNA-binding site, and a Dpp-dependent cyclic AMP-responsive element (Riese et al. 1997). The identification of response elements in the target genes $v e$ and $w g$ will be needed to investigate whether the regulation by Arm/Pan and Lin is direct and to reveal the precise mechanism at work. It is also possible that Lin interacts directly with Pan and/or Arm, rather than participating in a distinct complex. However, initial experiments with the two-hybrid system or immunoprecipitation have not provided evidence for direct interactions with Arm (V. Hatini and S. DiNardo, unpubl.).

In a second model, Lin may regulate transcription by creating a state permissive for Wg signal transducers to act. For example, Lin itself may act by remodeling chromatin structure, allowing efficient access of transcription factors to Wg-regulated promoters, leading to an increased activity of DNA-bound Pan/Arm complexes.
Finally, Lin may regulate transcription factor activity by affecting the modification state of factors such as Arm and or Pan. Precedent exists for this, as in Caenorhabditis elegans, the Lit1 MAP kinase regulates the function of Wrm1 and Pop1, proteins related to Arm and Pan, respectively (Ishitani et al. 1999; Meneghini et al. 1999; Rocheleau et al. 1999).

Although Lin can promote Wg signaling in the dorsal epidermis, it is not sufficient to do so ventrally. Thus, Lin can promote Wg signaling only in a competent region of cells. The activity of Lin dorsally must depend on additional factors, which provide this region with the competence to modulate $\mathrm{Wg}$ signaling in response to Lin. Such a factor could provide a biochemical link between Lin and the Arm/Pan complex.

Whichever model holds, note that the role of Arm and Pan is more clearly understood for transcriptional activation events (Molenaar et al. 1996; Brannon et al. 1997; Riese et al. 1997; van de Wetering et al. 1997), where Arm is proposed to confer an activation function to the otherwise negative regulator Pan (Cavallo et al. 1998; Roose et al. 1998; Waltzer and Bienz 1998). Only a few genes have been characterized as being repressed by Wg signal transduction, for example, $d p p$ and $w g$ in developing leg and wing, respectively, (Brook and Cohen 1996; Jiang and Struhl 1996; Rulifson et al. 1996), and ve as described here. Unfortunately, response elements mediating Wg-dependent repression have not yet been identified. It is likely that the Arm/Pan complex can recruit repressors or activators to $\mathrm{Wg}$ target genes, perhaps in a manner analogous to Smad proteins, which can activate by recruiting the co-activator $\mathrm{CBP} / \mathrm{p} 300$ (Feng et al. 1998) or repress by recruiting co-repressors such as TGIF or Ski, which, in turn, bind histone deacetylases (Akiyoshi et al. 1999; Luo et al. 1999; Wotton et al. 1999|. Our identification of $v e$ as a gene repressed by Wg input will eventually provide tools to investigate negative regulation by the Wg pathway.

\section{Regulation of Lin subcellular localization}

Lin can exhibit differential subcellular localization across the parasegment. Dorsally, when expressed at high levels, Lin is in nuclei of cells anterior to the En domain and in the cytoplasm of cells posterior to it. Thus, nuclear localization can correlate with cells that require lin function. Often one consequence of the activation of a signaling pathway is an accumulation of transcription factors and accessory proteins in the nucleus. This situation appears to be the case here, too, as the localization of Lin is dependent on Wg signaling as increased Wg signaling promotes Lin nuclear localization, while reduced Wg signaling leads to decreased nuclear localization in some regions in the embryo. In addition, Hh signaling, which is more active posterior to the En domain (Gritzan et al. 1999), may somehow promote Lin export from the nucleus. Irrespective of how signaling activity causes the differential localization, we note that Lin does not have an obvious nuclear localization signal. 
Because Lin is essential for Wg-dependent cell-type specification acting downstream or in parallel to Arm, and because Lin nuclear localization is influenced by $\mathrm{Wg}$, a particularly attractive model involves Arm in the localization of Lin. Arm/ $\beta$-catenin share homology with the importin/karyopherin family of transport receptors and can bind the nuclear pore machinery (Fagotto et al. 1998), raising the possibility that one function of Arm/ $\beta$-catenin is to import regulatory proteins to the nucleus. Perhaps as Wg signaling stabilizes Arm, it associates with Lin in the cytoplasm and transports Lin across the nuclear membrane. The association of Arm with other regulatory proteins may also promote their nuclear import, as proposed for Tsh (Gallet et al. 1998, 1999). Alternatively, because the phosphorylation state of a protein can lead to transport into or out of the nucleus, perhaps Wg signaling affects Lin localization through the inhibition of the serine/threonine kinase Shaggy. In this case, Lin nuclear import would be regulated by an upstream component of the Wg pathway and then interact with other downstream components (Arm/Pan) in the nucleus.

\section{Contribution of Lin to dorsal pattern}

Wg signaling has two distinct roles in patterning the embryonic epidermis. The first is to prevent an En-dependent signal from crossing over anteriorly (Heemskerk and DiNardo 1994; Gritzan et al. 1999; Sanson et al. 1999). When Wg signaling is deficient, a signal from the En domain, such as $\mathrm{Hh}$, crosses over and specifies cell fate anterior to it, creating a mirror image ve expression and cuticle pattern (smooth-1 $1^{\circ} 1^{\circ}$-smooth). In lin mutants, ve expression and pattern are similarly reorganized, indicating that lin is necessary for this role of $\mathrm{Wg}$. The second role of $\mathrm{Wg}$ is to specify cell fate in half of the parasegment, an event that is also blocked in lin mutants (Bokor and DiNardo 1996). Thus, these two patterning roles of $\mathrm{Wg}$ are compromised in lin mutants.

Normally, Hh patterns posteriorly from its source, specifying the $1^{\circ}-3^{\circ}$ cell fates, whereas Wg patterns to the anterior, specifying the $4^{\circ}$ cell fate. The domains of $\mathrm{Wg}$ and $\mathrm{Hh}$ influence meet in the middle of the parasegment, at approximately the interface of $3^{\circ}$ and $4^{\circ}$ cell types. A balance between $\mathrm{Wg}$ and $\mathrm{Hh}$ signaling is essential for proper patterning in this region as changing the strength of either can alter the pattern in the middle of the parasegment. For example, reduction of $\mathrm{Wg}$ function results in excess $3^{\circ}$ cells at the expense of $4^{\circ}$ cells (Fig. 1C). Similarly, a gradual increase in Hh signaling leads to a gradual increase in the number of $3^{\circ}$ cells at the expense of $4^{\circ}$ cells (Heemskerk and DiNardo 1994).

We propose that Lin helps govern the balance between $\mathrm{Wg}$ and $\mathrm{Hh}$ influence by promoting $\mathrm{Wg}$ signaling activity, which can thereby better compete with Hh function. An increase of Lin activity can alter this balance, leading to excess $4^{\circ}$ cells at the expense of $3^{\circ}$ cells (Fig. 3D). At higher levels of Lin, the entire domain under Hh influence differentiates as $4^{\circ}$ cells (Fig. 3C). Aside from the contribution of Lin levels to the pattern, the level of $\mathrm{Hh}$ is also crucial. Hh may contribute to this balance by antagonizing Lin function, as we proposed previously (Bokor and DiNardo 1996). The antagonism will be highest posterior to the En domain where Hh signaling is the highest. In fact, we now find further evidence for this antagonism, as expression of high levels of Lin in this region is not always sufficient to replace the $2^{\circ}$ with $4^{\circ}$ fate (Fig. 3C). We propose that, in wild type, Hh antagonizes Lin activity in the middle of the parasegment, thereby toning down $\mathrm{Wg}$ signaling activity in this region, and allowing these cells to adopt $3^{\circ}$ rather than $4^{\circ}$ fate. One way by which Hh may antagonize Lin function, and thereby Wg signaling, is by exporting Lin to the cytoplasm.

\section{Materials and methods}

\section{DNA manipulations}

lin fell under Df (2R)G75, narrowing the previous cytological position (Nüsslein-Volhard et al. 1984) to 44F-45A. By mobilizing a P-element inserted at the babo gene, we isolated an $\sim 40 \mathrm{kB}$ directed deletion, $\mathrm{Df}(2 \mathrm{R}) \mathrm{P} 15$, extending proximally from babo to $p g i$, which also deleted lin. DNA spanning this deletion was fully contained within cosmid 75F3 and P1 DS07727. We identified nine distinct cDNA classes isolated from a 4- to 8-hr embryonic library (Brown and Kafatos 1988). Of five overlapping genomic transgenes spanning the deletion, one (\#24) rescued lin mutants to adulthood. A smaller derivative transgene (\#24 KpnI $\mathrm{Cu}$ ) also rescued lin. Only one cDNA was encoded by this transgene. A 3.5-Kb HindIII-NotI cDNA fragment from pNB40 was inserted at the HindIII and NotI sites in pBluescript, generating pBS-Lin. Then, a KpnI-NotI blunted fragment from pBS-Lin was inserted at $\mathrm{KpnI}$ and blunted $\mathrm{XbaI}$ sites of pUAST vector.

For sequencing lin alleles, DNA was prepared from homozygous lin mutant larvae, recognized by their head open phenotype. Genomic DNA corresponding to nucleotides 29-945 of lin was PCR amplified from each of five lin alleles: $\operatorname{lin}^{\mathrm{G} 1}$ and $\operatorname{lin}{ }^{\mathrm{G} 2}$ (A. Konev), $\operatorname{lin}^{\mathrm{G} 10}$ (J. Merriam), $\operatorname{lin}^{2 \mathrm{f}}$, and $\operatorname{lin}^{2 \mathrm{u}}$ with forward primer 5'-CGCTGGCCCGAAGAGTGC-3' and reverse primer 5'-CTGGCCGTTGGTGTCCTGTGAGT-3'. Purified amplicons were sequenced using these PCR primers and two internal primers: forward primer, 5'-CCCCACCACCACGCGGTGTAG3'(622-643); reverse primer, 5'-TTGGCGGTGCTTGGCGTTGAC-3' (615-594).

For generating genomic Myc-tagged lin transgenes, we PCR amplified a region of the lin cDNA with a foward primer upstream of the EcoRI site at position 2380 and a reverse primer spanning the termination codon at position 2867, introducing a BamHI site: forward primer, 5' -AGCGGTGACGGTGAAAGAGATACG-3'; reverse primer, 5'-CGGGATCCGCTCAGCTCGTTGCCCTCGTACAG-3' (BamHI site underlined). The amplicon was digested with EcoRI and BamHI and cloned into pBluescript SK. Then, a BamHI-XbaI fragment from pCS2$6 \times \mathrm{MT}$ was inserted into the above construct, fusing the carboxyl terminus of Lin to the $6 x-M y c$ epitope. Then an XhoI fragment extending from position 2840 of lin past the $6 \times-$ Myc epitope was isolated and inserted at the unique XhoI site in \#24 $K p n I \mathrm{Cu}$, corresponding to position 2840 in the lin cDNA, generating a Lin-Myc tag fusion protein. The orientation and frame of the construct was verified by sequencing.

For generation of the bacterial Lin protein expression vector, after conversion of the SmaI site at position 596 in the lin cDNA to a BamHI site, a BamHI-Xhol fragment (corresponding to nucleotides 596-2824; amino acids 104-843) was inserted 
into BamHI/SalI-digested pQE-32, in-frame with an amino-terminal 6×His epitope (Qiagen).

\section{Antibody production}

The induced Lin protein accumulated in inclusion bodies, which were purified by four cycles of resuspension, sonication, and precipitation and prepared for immunization by Covance, Inc., as described by Benedyk (1994). Antibodies where purified by adsorption to membrane-bound Lin protein as described by Pringle et al. (1991)

\section{Fly strains}

Five independent transgenic lines carrying UAS-Lin showed comparable effects on cuticle pattern. Two of these were used here: UAS-Lin 8 (chromosome II); UAS-Lin 9/2 (chromosome III). We used Wg-GAL4 (J. Pradel), En-GAL4 (A. Brand), PtcGAL4 (Speicher et al. 1994), Arm-GAL4 (J.-P. Vincent), UAS$\operatorname{Pan}^{\mathrm{DN1}}$ (van de Wetering et al. 1997), UAS-Arm ${ }^{\text {S10 }}$ (Pai et al. 1997), UAS-LacZ.NZ J312 (Y. Hiromi), and UAS-Wg (Lawrence et al. 1996). We constructed the following recombinant chromosomes: $\operatorname{lin}^{2 \mathrm{f}}$ En-GAL4, $\operatorname{lin}^{2 \mathrm{f}}$ Ptc-GAL4, $\operatorname{lin}^{2 \mathrm{f}}$ UAS-Wg, FRT42B $\operatorname{lin}^{\mathrm{G} 12-30}$, and FRT42B $\operatorname{lin}^{2 \mathrm{u}}$. Germ-line clones for $\operatorname{lin}^{\mathrm{G} 12-30}$ and $\operatorname{lin}^{2 \mathrm{u}}$ were produced by use of the $O V O^{\mathrm{D} 1}$ system (Chou et al. 1993) in females of the genotype yw HSFlp/yw; FRT42B $\operatorname{lin}^{\mathrm{G} 12-30}$ or FRT42B $\operatorname{lin}^{2 \mathrm{u}} / \mathrm{FRT} 42 \mathrm{~B}$ ovo ${ }^{\mathrm{D} 1}$, which were mated to yw; $\operatorname{lin}^{2 \mathrm{f}} / \mathrm{CyO} \mathrm{Df}(2 \mathrm{R}) \mathrm{B} 80 \mathrm{Dp}$ y+ males. Embryos mutants for both maternal and zygotic lin function, FRT42D linmat/zyg $/ \operatorname{lin}^{2 f}$, were identified by their y cuticle phenotype.

\section{Genotypes analyzed}

The following genotypes were analyzed in this study: $h h^{\text {ts2 }}$ (Fig. 1B); Ptc-GAL4; UAS-Pan ${ }^{\mathrm{DN} 1}$ (Figs. 1C, 5B, 6B, and 7D); Df (2R)P15 (Figs. 1D, 5C, and 7A); $\operatorname{lin}^{\mathrm{G} 2} / \operatorname{lin}^{2 \mathrm{f}}$; \#24 KpnI Cu (Fig. $2 \mathrm{~A}) ; \operatorname{lin}^{\mathrm{G} 2} / \operatorname{lin}^{2 \mathrm{f}}$ (Fig. 2C); $\operatorname{lin}^{2 \mathrm{f}}$ Ptc-GAL4/ $\operatorname{lin}^{\mathrm{G} 2}$; UAS-Lin $9 / 2$ (Fig. 2D); Ptc-GAL4; UAS-Arm ${ }^{\mathrm{S} 10}$ (Figs. 3B, 5E, 6C, and 7B); UAS-Lin 9/2/Arm-GAL4 (Fig. 3C); Ptc-GAL4; UAS-Lin 9/2 (Figs. 3D, 5F, and 7E); lin $^{2 \mathrm{f}} \mathrm{En}-\mathrm{Gal} 4 /$ lin $^{\mathrm{G} 2}$; UAS-LacZ.NZ (Fig. 4A); $\operatorname{lin}^{2 \mathrm{f}}$ En-GAL4/ $\operatorname{lin}^{\mathrm{G} 2}$; UAS-Lin 9/2 (Fig. 4B); $\operatorname{lin}^{\mathrm{G} 2} \mathrm{Wg}-$ GAL4//in ${ }^{2 \mathrm{f}}$; UAS-Lin 9/2 (Fig. 4C); $\operatorname{lin}^{2 \mathrm{f}}$ (Fig. 6D); UAS-Arm ${ }^{\mathrm{S1O}}$; $\operatorname{lin}^{2 \mathrm{f}} / \operatorname{lin}^{2 \mathrm{f}}$ Ptc-GAL4 (Figs. 6E and 7C); Ptc-GAL4/ UAS-Lin 8; UAS-Pan $^{\text {DN1 }}$ (Figs. $7 \mathrm{~F}$ and $8 \mathrm{C}$ ); Ptc-GAL4/ UAS-Wg; UAS-Lin 9/2 (Fig. 8B).

Cuticle preparations, immunohistochemistry, and in situ hybridization

Embryos were processed as described previously to visualize cuticle pattern (van der Meer 1977; Wieschaus and NüssleinVolhard 1986). $\beta$-Galactosidase activity stains on cuticle preparations (Heemskerk and DiNardo 1994) were performed on 1 in $^{2 f}$ En-Gal4/ lin ${ }^{\mathrm{G} 2}$; UAS-LacZ.NZ. We used MAb4D4 for visualization of Wg (Brook and Cohen 1996), MAb4D9 for En (Patel et al. 1989), MAb9E10 for the Myc epitope, and crude or purified rat serum for Lin. Several fixation protocols were used to detect Lin in situ in wild-type embryos, including variation of the duration and fixative concentrations, heat fixation, and the use of octane instead of methanol. Biotinylated antibodies (Vector) and horseradish peroxidase-conjugated streptavidin were used at 1:400. Cy3-labeled secondary antibodies were used at 1:300 (Jackson). In situ probes were synthesized from full-length lin cDNA and from ve and $w g$ templates (Baker 1987; Bier et al. 1990). Double labeling was as described by Dougan and Di-
Nardo (1992). Embryos were photographed as whole mount or as sections.

\section{Acknowledgments}

We thank B. Wilder and members of our laboratory for helpful discussions. We also thank M. Jiang for excellent technical assistance. We are indebted to J. Merriam for help initially with the identification of nearby $\mathrm{P}$ elements, for graciously hosting one of us (P.B.) for an extended visit, and for lines alleles. We also thank A. Konev for lines alleles, S. Wasserman for help interpreting P-element-induced genomic rearrangements, and L. Marsh for friendly exchanges of information early on. This work was supported by National Institutes of Health grant GM 45747 to S.D. V.H. is supported by a fellowship from the Cancer Research Fund of the Damon Runyon-Walter Winchell Foundation, DRG-1442.

The publication costs of this article were defrayed in part by payment of page charges. This article must therefore be hereby marked "advertisement" in accordance with 18 USC section 1734 solely to indicate this fact.

\section{References}

Akiyoshi, S., H. Inoue, J. Hanai, K. Kusanagi, N. Nemoto, K. Miyazono, and M. Kawabata. 1999. c-Ski acts as a transcriptional co-repressor in transforming growth factor-beta signaling through interaction with smads. I. Biol. Chem. 274: 35269-35277.

Alexandre, C., M. Lecoutois, and J.-P. Vincent. 1999. Wingless and Hedgehog pattern Drosophila denticle belts by regulating the production of short-range signals. Development 126: 5689-5698.

Baker, N.E. 1987. Molecular cloning of sequences from wingless a segment polarity gene in Drosophila: The spatial distribution of a transcript in embryos. EMBO J. 6: 1765-1773.

. 1988. Embryonic and imaginal requirements for $w g$, a segment polarity gene in Drosophila. Dev. Biol. 125: 96-108.

Behrens, J., J.P. von Kries, M. Kuhl, L. Bruhn, D. Wedlich, R. Grosschedl, and W. Birchmeier. 1996. Functional interaction of $\beta$-catenin with the transcription factor LEF-1. Nature 382: 638-642.

Bejsovec, A. and A. Martinez-Arias. 1991. Roles of wingless in patterning the larval epidermis of Drosophila. Development 113: 471-485.

Benedyk, M.J., J.R. Mullen, and S. DiNardo. 1994. odd-paired: A zinc finger pair-rule protein required for the timely activation of engrailed and wingless in Drosophila embryos. Genes \& Dev. 8: 105-117.

Bhanot, P., M. Fish, J.A. Jemison, R. Nusse, J. Nathans, and K.M. Cadigan. 1999. Frizzled and Dfrizzled-2 function as redundant receptors for Wingless during Drosophila embryonic development. Development 126: 4175-4186.

Bhat, K.M. 1998. frizzled and frizzled 2 play a partially redundant role in wingless signaling and have similar requirements to wingless in neurogenesis. Cell 95: 1027-1036.

Bier, E., L.Y. Jan, and Y.N. Jan. 1990. Rhomboid a gene required for dorsoventral axis establishment and peripheral nervous system development in Drosophila melanogaster. Genes \& Dev. 4: 190-203.

Bokor, P. and S. DiNardo. 1996. The roles of hedgehog, wingless and lines in patterning the dorsal epidermis in Drosophila. Development 122: 1083-1092.

Brannon, M., M. Gomperts, L. Sumoy, R.T. Moon, and D. 
Kimelman. 1997. A $\beta$-catenin/XTcf-3 complex binds to the siamois promoter to regulate dorsal axis specification in Xenopus. Genes \& Dev. 11: 2359-2370.

Brook, W.J. and S.M. Cohen. 1996. Antagonistic interactions between wingless and decapentaplegic responsible for dorsal-ventral pattern in the Drosophila Leg. Science 273: 13731377.

Brown, N.H. and F.C. Kafatos. 1988. Functional cDNA libraries from Drosophila embryos. J. Mol. Biol. 203: 425-437.

Brunner, E., O. Peter, L. Schweizer, and K. Basler. 1997. pangolin encodes a Lef- 1 homologue that acts downstream of armadillo to transduce the Wingless signal in Drosophila. Nature 385: 829-833.

Cabrera, C.V., M.C. Alonso, P. Johnston, R.G. Phillips, and P.A. Lawrence. 1987. Phenocopies induced with antisense RNA identify the wingless gene. Cell 50: 659-663.

Castelli-Gair, J. 1998. The lines gene of Drosophila is required for specific functions of the Abdominal-B HOX protein. Development 125: 1269-1274.

Cavallo, R.A., R.T. Cox, M.M. Moline, J. Roose, G.A. Polevoy, H. Clevers, M. Peifer, and A. Bejsovec. 1998. Drosophila Tcf and Groucho interact to repress Wingless signalling activity. Nature 395: 604-608.

Chen, C. and G. Struhl. 1999. Wingless transduction by the frizzled and frizzled2 proteins of drosophila. Development 126: $5441-5452$.

Chou, T.B., E. Noll, and N. Perrimon. 1993. Autosomal P[ovoD1] dominant female-sterile insertions in Drosophila and their use in generating germ-line chimeras. Development 119: 1359-1369.

de Zulueta, P., E. Alexandre, B. Jacq, and S. Kerridge. 1994. Homeotic complex and teashirt genes co-operate to establish trunk segmental identities in Drosophila. Development 120: $2287-2296$

Dierick, H. and A. Bejsovec. 1999. Cellular mechanisms of wingless/Wnt signal transduction. Curr. Top. Dev. Biol. 43: 153-190.

DiNardo, S., E. Sher, J. Heemskerk-Jongens, J.A. Kassis, and P.H. O'Farrell. 1988. Two-tiered regulation of spatially patterned engrailed gene expression during drosophila embryogenesis. Nature 332: 604-609.

Dougan, S.T. and S. DiNardo. 1992. wingless generates cell type diversity among engrailed expressing cells. Nature 360: $347-$ 350.

Fagotto, F., U. Gluck, and B.M. Gumbiner. 1998. Nuclear localization signal-independent and importin/karyopherin-independent nuclear import of beta-catenin. Curr. Biol. 8: 181190.

Feng, X.H., Y. Zhang, R.Y. Wu, and R. Derynck. 1998. The tumor suppressor Smad4/DPC4 and transcriptional adaptor $\mathrm{CBP} / \mathrm{p} 300$ are coactivators for $\operatorname{smad} 3$ in TGF- $\beta$-induced transcriptional activation. Genes \& Dev. 12: 2153-2163.

Gallet, A., C. Angelats, A. Erkner, B. Charroux, L. Fasano, and S. Kerridge. 1999. The C-terminal domain of armadillo binds to hypophosphorylated teashirt to modulate wingless signalling in Drosophila. EMBO J. 18: 2208-2217.

Gallet, A., A. Erkner, B. Charroux, L. Fasano, and S. Kerridge. 1998. Trunk-specific modulation of wingless signalling in Drosophila by teashirt binding to armadillo. Curr. Biol. 8: 893-902.

Giese, K., J. Cox, and R. Grosschedl. 1992. The HMG domain of lymphoid enhancer factor 1 bends DNA and facilitates assembly of functional nucleoprotein structures. Cell 69: 185195.

Gritzan, U., V. Hatini, and S. DiNardo. 1999. Mutual antagonism between signals secreted by adjacent wingless and en- grailed cells leads to specification of complementary regions of the Drosophila parasegment. Development 126: 41074115.

Heemskerk, J. and S. DiNardo. 1994. Drosophila hedgehog acts as a morphogen in cellular patterning. Cell 76: 449-460.

Ishitani, T., J. Ninomiya-Tsuji, S. Nagai, M. Nishita, M. Meneghini, N. Barker, M. Waterman, B. Bowerman, H. Clevers, H. Shibuya, and K. Matsumoto. 1999. The TAK1-NLKMAPK-related pathway antagonizes signalling between $\beta$-catenin and transcription factor TCF. Nature 399: 798802.

Jiang, J. and G. Struhl. 1996. Complementary and mutually exclusive activities of decapentaplegic and wingless organize axial patterning during Drosophila leg development. Cell 86: 401-409.

Kennerdell, J.R. and R.W. Carthew. 1998. Use of dsRNA-mediated genetic interference to demonstrate that frizzled and frizzled 2 act in the wingless pathway. Cell 95: 1017-1026.

Lawrence, P.A., B. Sanson, and J.P. Vincent. 1996. Compartments, wingless and engrailed: Patterning the ventral epidermis of Drosophila embryos. Development 122: 4095-4103.

Luo, K., S.L. Stroschein, W. Wang, D. Chen, E. Martens, S. Zhou, and Q. Zhou. 1999. The Ski oncoprotein interacts with the Smad proteins to repress TGFbeta signaling. Genes \& Dev. 13: 2196-2206.

Martinez-Arias, A., N.E. Baker, and P.W. Ingham. 1988. Role of segment polarity genes in the definition and maintenance of cell states in the Drosophila embryo. Development 103: 157-170.

Meneghini, M.D., T. Ishitani, J.C. Carter, N. Hisamoto, J. Ninomiya-Tsuji, C.J. Thorpe, D.R. Hamill, K. Matsumoto, and B. Bowerman. 1999. MAP kinase and Wnt pathways converge to downregulate an HMG-domain repressor in Caenorhabditis elegans. Nature 399: 793-797.

Molenaar, M., M. van de Wetering, M. Oosterwegel, J. PetersonMaduro, S. Godsave, V. Korinek, J. Roose, O. Destree, and H. Clevers. 1996. XTcf-3 transcription factor mediates $\beta$-catenin-induced axis formation in Xenopus embryos. Cell 86: 391-399.

Muller, H., R. Samanta, and E. Wieschaus. 1999. Wingless signaling in the Drosophila embryo: Zygotic requirements and the role of the frizzled genes. Development 126: 577-586.

Noordermeer, J., P. Johnston, F. Rijsewik, R. Nusse, and P.A. Lawrence. 1992. The consequences of ubiquitous expression of the wingless gene in the Drosophila embryo. Development 116: 711-719.

Nüsslein-Volhard, C. and E. Wieschaus. 1980. Mutations affecting segment number and polarity in Drosophila. Nature 287: 795-801

Nüsslein-Volhard, C., E. Wieschaus, and H. Kluding. 1984. Mutations affecting the pattern of the larval cuticle in Drosophila melanogaster. I. Zygotic loci on the second chromosome. Roux's Arch. Dev. Biol. 193: 267-282.

O' Keefe, L., S.T. Dougan, L. Gabay, E. Raz, B.Z. Shilo, and S. DiNardo. 1997. Spitz and Wingless, emanating from distinct borders, cooperate to establish cell fate across the Engrailed domain in the Drosophila epidermis. Development 124: $4837-4845$

Pai, L.M., S. Orsulic, A. Bejsovec, and M. Peifer. 1997. Negative regulation of Armadillo, a Wingless effector in Drosophila. Development 124: 2255-2266.

Patel, N.H., E. Martin-Blanco, K.G. Coleman, S.J. Poole, M.C. Ellis, T.B. Kornberg, and C.S. Goodman. 1989. Expression of engrailed proteins in arthropods, annelids, and chordates. Cell 58: 955-968.

Payre, F., A. Vincent, and S. Carreno. 1999. ovo/svb integrates 
Hatini et al.

Wingless and DER pathways to control epidermis differentiation. Nature 400: 271-275.

Pringle, J., A. Adams, D. Drubin, and B. Haaker. 1991. Immunofluorescence methods for yeast. In Guide to yeast genetics and molecular biology (ed. C. Guthrie and G. Fink), pp. 581584.

Riese, J., X. Yu, A. Munnerlyn, S. Eresh, S.C. Hsu, R. Grosschedl, and M. Bienz. 1997. LEF-1, a nuclear factor coordinating signaling inputs from wingless and decapentaplegic. Cell 88: 777-787.

Rijsewijk, F., M. Scheurmann, E. Wagenaar, P. Parren, D. Weigel, and R. Nusse. 1987. The Drosophila homolog of the mouse mammary oncogene int-1 is identical to the segment polarity gene wingless. Cell 50: 649-657.

Rocheleau, C.E., J. Yasuda, T.H. Shin, R. Lin, H. Sawa, H. Okano, J.R. Priess, R.J. Davis, and C.C. Mello. 1999. WRM-1 activates the LIT-1 protein kinase to transduce anterior/posterior polarity signals in C. elegans. Cell 97: 717-726.

Roose, J., M. Molenaar, J. Peterson, J. Hurenkamp, H. Brantjes, P. Moerer, M. van de Wetering, O. Destree, and H. Clevers. 1998. The Xenopus Wnt effector XTcf-3 interacts with Groucho-related transcriptional repressors. Nature 395: 608-612.

Rulifson, E.J., C.A. Micchelli, J.D. Axelrod, N. Perrimon, and S.S. Blair. 1996. wingless refines its own expression domain on the Drosophila wing margin. Nature 384: 72-74.

Sanson, B., C. Alexandre, N. Fascetti, and J.-P. Vincent. 1999. Engrailed and Hedgehog make the range of Wingless asymmetric in Drosophila embryos. Cell 98: 207-216.

Speicher, S.A., U. Thomas, U. Hinz, and E. Knust. 1994. The Serrate locus of Drosophila and its role in morphogenesis of the wing imaginal discs: Control of cell proliferation. Development 120: 535-544.

Spemann. 1938. Embryonic development and induction. Yale University Press, New Haven, CT.

Szüts, D., M. Freeman, and M. Bienz. 1997. Antagonism between EGFR and Wingless signaling in the larval cuticle of Drosophila. Development 124:

Tabata, T., S. Eaton, and T.B. Kornberg. 1992. The Drosophila hedgehog gene is expressed specifically in posterior compartment cells and is a target of engrailed regulation. Genes \& Dev. 6: 2635-2645.

van de Wetering, M., R. Cavallo, D. Dooijes, M. van Beest, J. van Es, J. Loureiro, A. Ypma, D. Hursh, T. Jones, A. Bejsovec, M. Peifer, M. Mortin, and H. Clevers. 1997. Armadillo coactivates transcription driven by the product of the Drosophila segment polarity gene dTCF. Cell 88: 789-799.

van den Heuvel, M., J. Klingensmith, N. Perrimon, and R. Nusse. 1993. Cell patterning in the Drosophila segment: Engrailed and wingless antigen distributions in segment polarity mutant embryos. Development (Suppl.) :105-114.

van der Meer, S. 1977. Optical clean and permanent whole mount preparation for phase contrast microscopy of cuticular structures of insect larvae. Drosophila Inf. Serv. 52: 160161.

Waltzer, L. and M. Bienz. 1998. Drosophila CBP represses the transcription factor TCF to antagonize Wingless signalling. Nature 395: 521-525.

Wiellette, E.L. and W. McGinnis. 1999. Hox genes differentially regulate Serrate to generate segment-specific structures. Development 126: 1985-1995.

Wieschaus, E. and C. Nüsslein-Volhard. 1986. Looking at embryos in Drosophila: A practical approach. pp. 199-227. IRL Press, Oxford, UK.

Wotton, D., R.S. Lo, S. Lee, and J. Massagué. 1999. A Smad transcriptional corepressor. Cell 97: 29-39. 


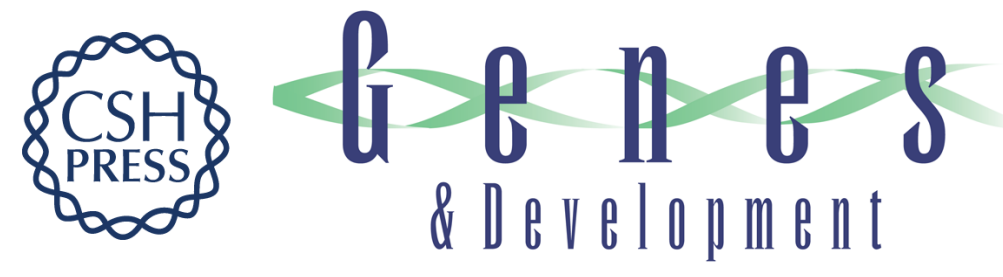

\section{Tissue- and stage-specific modulation of Wingless signaling by the segment polarity gene lines}

Victor Hatini, Peter Bokor, Ryoko Goto-Mandeville, et al.

Genes Dev. 2000, 14:

Access the most recent version at doi:10.1101/gad.14.11.1364

References

This article cites 60 articles, 26 of which can be accessed free at: http://genesdev.cshlp.org/content/14/11/1364.full.html\#ref-list-1

License

Email Alerting

Receive free email alerts when new articles cite this article - sign up in the box at the top Service right corner of the article or click here.

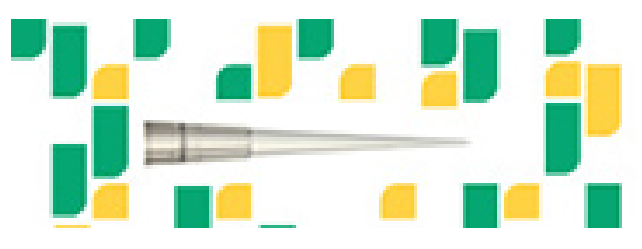

Focused on your science. 